\title{
Improvement of symptoms in patients with sick sinus syndrome by spontaneous development of stable atrial fibrillation ${ }^{1,2}$
}

\author{
ZAKAUDDIN VERA, DEAN T. MASON, NAJAM A. AWAN, \\ RICHARD R. MILLER, DAVID JANZEN, MELVIN J. TONKON, AND \\ LOUIS A. VISMARA \\ From Section of Cardiovascular Medicine, Departments of Medicine and Physiology, \\ University of California at Davis, School of Medicine and Sacramento Medical Center, \\ Davis and Sacramento, California, U.S.A.
}

Fifty-six patients with symptomatic chronic sinus bradycardia because of sick sinus syndrome (SSS) were followed for periods from one month to 11 years (average $3 \cdot 2$ years). Eleven developed stable atrial fibrillation persisting for 8 to 61 months; 52 had permanent demand pacemakers implanted before atrial fibrillation commenced. In the 11 patients with atrial fibrillation, 10 had adequate ventricular rate, 8 with rates $>100$ beats/min requiring digoxin for rate control. The 8 patients with atrial fibrillation with pacemakers remained asymptomatic for 13 to 18 months without requiring reimplantation; battery failure occurred in 2 whose rapid ventricular rates were controlled by digoxin. In the other 6 patients with pacemakers who developed atrial fibrillation, adequate ventricular rates persisted resulting in overdrive suppression. No patient had systemic embolisation. The previous duration of symptomatic sinus bradycardia was longer in patients developing atrial fibrillation (average 5.5 years) compared $(P<0.01)$ with patients without atrial fibrillation $(1.9$ years). Further, premature atrial contractions occurred in all 11 patients before atrial fibrillation in contrast to only 21 of the 45 patients without atrial fibrillation. It is concluded that occurrence of atrial fibrillation in SSS with symptomatic sinus bradycardia provides a natural cure of symptoms caused by bradycardia. These data indicate that permanent ventricular pacing may not be necessary if persistent atrial fibrillation develops in SSS.

Symptomatic chronic sinus bradycardia in the sick sinus syndrome (SSS) is generally refractory to long-term pharmacological therapy, and in most instances permanent pacemakers are required for the relief of symptoms (Ferrer, 1973). While many aspects of the natural history of this disorder are not well established, it is recognised that sinus node function worsens with time and lifelong pacemaker treatment is, therefore, anticipated. Some patients with SSS are prone to episodes of supra-ventricular tachyarrhythmias including paroxysmal atrial fibrillation; alternating sinus bradycardia and tachy-

${ }^{1}$ Presented in part at the 48th Annual Scientific Sessions of the American Heart Association, 19 November 1975, Anaheim, California, U.S.A.

${ }^{2}$ Supported in part by a research grant from the American Heart Association; and a Research Program Project Grant and Postgraduate Training Grant from the National Heart and Lung Institute, National Institutes of Fealth, Bethesda, Maryland, U.S.A.

Received for publication 19 July 1976 cardia, usually supraventricular, is termed the 'bradycardia-tachycardia syndrome' (Short, 1954). The occurrence of transient atrial fibrillation in patients with SSS provides the intriguing possibility that spontaneous or induced stable atrial fibrillation may offer a natural therapeutic mechanism for maintaining an accelerated ventricular response in this entity, thus obviating the need for permanent pacemaker implantation. Indeed, as early as 1954 Short observed that one of his patients with the 'bradycardia-tachycardia syndrome' became asymptomatic upon developing atrial fibrillation and, furthermore, imaginatively suggested that a means for inducing atrial fibrillation in these patients would be of therapeutic value (Short, 1954). Though stable atrial fibrillation has been observed in SSS (Ferrer, 1973; Rubenstein et al., 1972; Krishnaswami and Geraci, 1975), the clinical importance of atrial fibrillation 
in this setting has not been fully realieds. The circumstances, incidence, and consequences relative to the development of persistent atrial fibrillation in SSS are unknown. The present investigation was, therefore, carried out over an 11-year period at our institution in a population of 56 patients with SSS in whom stable atrial fibrillation developed in 11 patients. In this study the clinical features of SSS patients with and without the development of atrial fibrillation are compared.

\section{Methods and results}

Fifty-six patients ( 35 men and 21 women) with electrocardiographic evidence of chronic symptomatic inappropriately slow sinus rates which were either periodic or persistent form the basis of this study. Their ages ranged from 54 to 90 years (average 61 years). The duration of follow-up was from 1 month to 11 years. The severity of the symptoms caused by slow heart rate in 53 patients necessitated insertion of permanent demand ventricular pacemakers whereas in 3 they did not. In all of the 56 patients sinus bradycardia resulted in a variety of symptoms ranging from lethargy and tiredness to frank syncopal episodes. The slow sinus rates were not related to drug therapy including digitalis, propranolol, or reserpine. In addition their heart rates showed inadequate increases after administration of intravenous atropine (Ferrer, 1973). Of the 53 patients, 31 also underwent rapid atrial stimulation for the evaluation of sinus node recovery time (Mandel et al., 1971).

Fig. 1, of patient H.W. with syncopal episodes, exemplifies a severe form of sinus bradycardia in whom the heart rate was only $20 \mathrm{bpm}$ during syncope. The lack of a consistent escape mechanism from the atria, AV junction, or lower pacemaker sites contributes to the genesis of cardiac asystole. This is a frequent finding in SSS and appears to indicate associated disease of sub-sinoatrial nodal structures with depressed automaticity. Fig. 2 shows an example of a very abnormal prolongation of sinus node recovery time in patient R.H. with symptomatic sinus bradycardia. The top panel showed slow sinus rhythm at a rate of $56 \mathrm{bpm}$. In the bottom tracing the right atrium was paced at $98 \mathrm{bpm}$; after abrupt cessation of atrial pacing, the sinus node required the extraordinarily long recovery period of $3.26 \mathrm{~s}$ to generate a sinus beat.

During the course of follow-up of the 53 patients with persistent SSS, 8 developed atrial fibrillation which has remained stable for 8 to 38 months. Three additional patients presented with chronic atrial fibrillation who, after undergoing electrical cardioversion, emerged with extremely slow sinus or junctional rhythm. In each of these 3 subjects, there was historical and previous electrocardiographic evidence of pronounced symptomatic sinus bradycardia, thereby affording the diagnosis of SSS in retrospect. All 3 patients spontaneously reverted to atrial fibrillation. The pertinent clinical in-

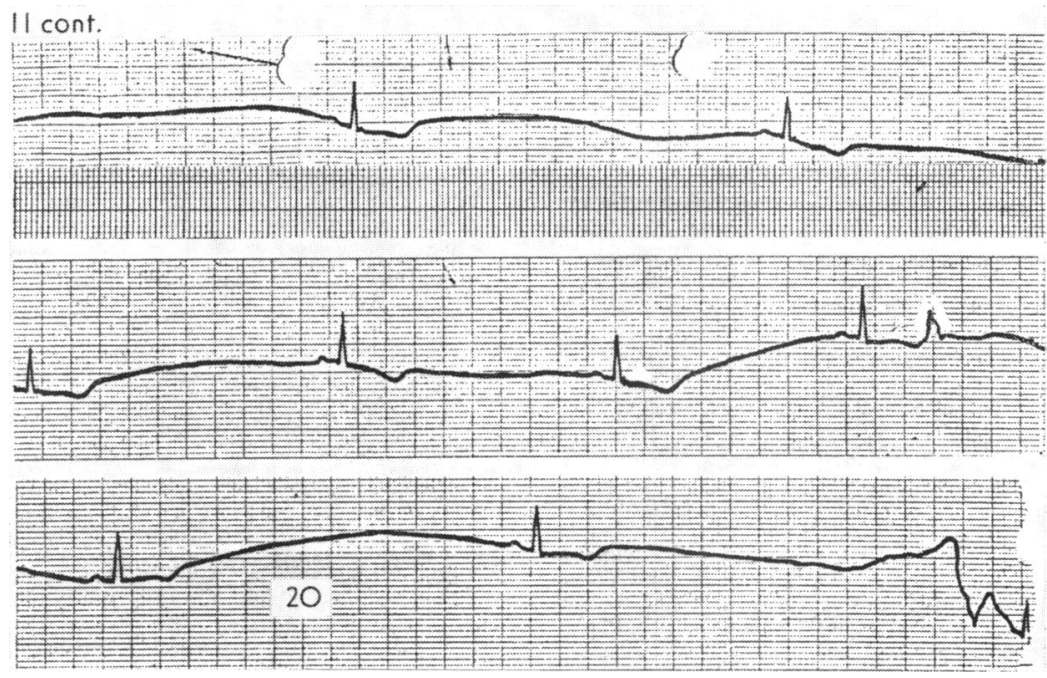

Fig. 1 Electrocardiogram (lead II) recorded during a period of syncope in patient H.W. with the sick sinus syndrome. The three continuous strips show severe sinus bradyarrhythmia of 20 beats/min with a premature ectopic ventricular beat. The lack of an escape mechanism between the infrequent sinus beats is noteworthy. 


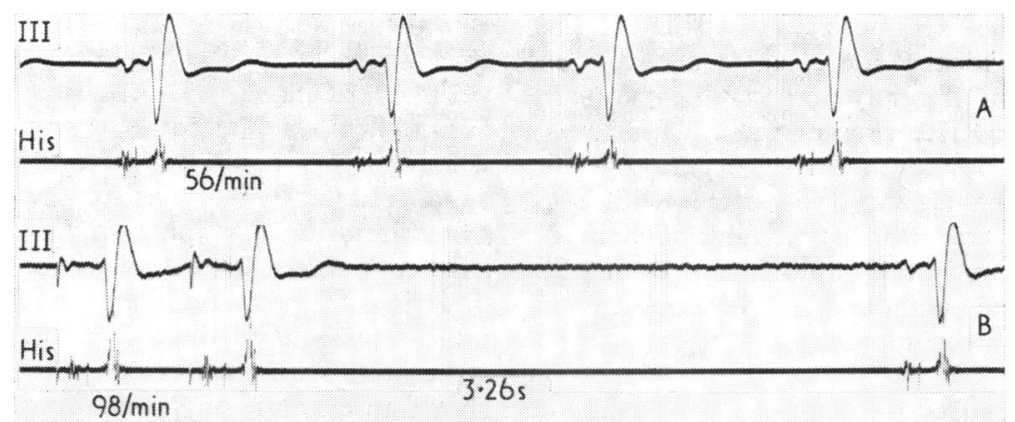

Fig. 2 Representative tracing of Lead III and the His bundle electrogram showing the sinus node recovery time in $S S S$ patient R.H. The top panel (A) shows sinus bradycardia (rate 56/min). In the bottom panel (B) right atrial pacing was carried out at a rate of $98 / \mathrm{min}$. Abrupt cessation of pacing was followed by a very long pause of $3 \cdot 26$ during which no escape activity was manifested.

formation in the 11 patients with SSS and stable atrial fibrillation is presented in Table 1 . Selected patients below delineate the characteristic features and modes of presentation in SSS with persistent atrial fibrillation.

\section{(1) SICK SINUS SYNDROME PRESENTING} WITH CHRONIC ATRIAL FIBRILLATION A 67-year-old woman (Case 1, Table 1) presented with atrial fibrillation and a moderately rapid resting ventricular response of $90 \mathrm{bpm}$ (Fig. 3). Her only symptoms consisted of occasional palpitation. Elective cardioversion was carried out; the immediate postcardioversion period showed very slow sinus rate with erratic atrial activity and periods of junctional escape rhythm as shown by the continuous recordings $\mathrm{A}$ through $\mathrm{E}$ in Fig. 3. The

Table 1 SSS with stable atrial fibrillation

\begin{tabular}{|c|c|c|c|c|c|c|c|}
\hline $\begin{array}{l}\text { Case } \\
\text { No. }\end{array}$ & $\begin{array}{l}\text { Age } \\
(y)\end{array}$ & Sex & $\begin{array}{l}\text { SSS } \\
\text { duration } \\
\text { before } \\
A F(y)\end{array}$ & $\begin{array}{l}\text { Duration } \\
\text { of } A F \\
\text { (mth) }\end{array}$ & $\begin{array}{l}\text { Ventri- } \\
\text { cular } \\
\text { response } \\
\text { during } \\
A F\end{array}$ & $\begin{array}{l}A P B \\
\text { before } \\
A F\end{array}$ & $\begin{array}{l}\text { Associated } \\
\text { cardiac } \\
\text { disease }\end{array}$ \\
\hline 1 & 67 & $\mathbf{F}$ & 5 & 61 & 90 & + & None \\
\hline 2 & 69 & $\mathbf{M}$ & 5 & 24 & 160 & + & CAD \\
\hline 3 & 77 & $\mathbf{M}$ & 5 & 20 & 105 & + & None \\
\hline 4 & 70 & $\mathbf{M}$ & 11 & 18 & 120 & + & None \\
\hline & 67 & $\mathbf{F}$ & 4 & 8 & 110 & + & \\
\hline 6 & 82 & M & 6 & 8 & 100 & + & CAD \\
\hline 7 & 66 & $\mathbf{F}$ & 3 & 25 & 140 & + & $\begin{array}{l}\text { CAD, } \\
\text { HCVD }\end{array}$ \\
\hline 8 & 83 & F & 8 & 38 & 30 & + & HCVD \\
\hline 9 & 80 & $\mathbf{M}$ & 5 & 13 & 90 & + & CAD \\
\hline 10 & 65 & $\mathbf{M}$ & 3 & 10 & 100 & t & COPD \\
\hline 11 & 61 & $\mathbf{M}$ & 5 & 12 & 100 & + & None \\
\hline
\end{tabular}

Abbreviations: AF, stable atrial fibrillation; APB, atrial premature beats; + , presence of APB before AF; CAD, coronary arterial disease; AS, congenital aortic stenosis; HCVD, essential hypertension; COPD, chronic lung disease. slowest ventricular response was $34 \mathrm{bpm}$ in strips $A$ and B. Her bradycardia responded poorly $(<70 \mathrm{bpm}$ ) to $1.5 \mathrm{mg}$ intravenous atropine. During intensive care unit monitoring atrial fibrillation returned within a few hours, and this arrhythmia has remained stable with adequate ventricular response to date. Review of previous electrocardiograms taken elsewhere pointed to atrial fibrillation of at least three months' duration and pronounced sinus bradycardia with periods of sinus arrest for 3 years before onset of atrial fibrillation. In addition, before atrial fibrillation, she had had dizzy spells

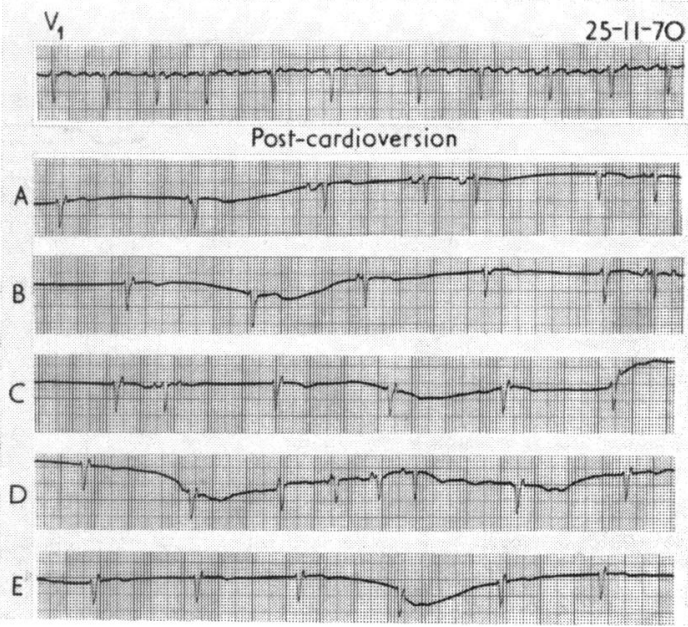

Fig. 3 Representative lead $V 1$ recording of the emergence of premature atrial beats and escape junctional rhythm (continuous panels $A$ through $E$ ) in Case 1 after elective cardioversion of chronic atrial fibrillation (shown in the top panel). 
and periodic weakness for 2 years suggesting SSS for this prolonged period.

Two further patients (Cases 2 and 3, Table 1) presented similarly with atrial fibrillation and after cardioversion had slow sinus rates. In both of these patients, reversion to chronic atrial fibrillation occurred which has persisted to the present time.

\section{(2) SSS WITH PERMANENT PACEMAKERS} DEVELOPING STABLE ATRIAL FIBRILLATION AND ADEQUATE VENTRICULAR RESPONSE Eight of the remaining patients with SSS who developed chronic atrial fibrillation had had permanent pacemakers implanted. In two of these patients (Cases 4 and 9, Table 1) battery failure occurred. However, in view of the stable atrial fibrillation with an adequate heart rate, shown by serial electrocardiograms and two 24-hour periods of ambulatory electrocardiographic monitoring, and the lack of symptoms, pacemaker replacement was not recommended in either patient. Subsequently, both patients remained asymptomatic with an adequate ventricular response in the presence of persistent atrial fibrillation.

Case 4, a 70-year-old man, had had syncope caused by SSS for 4 years before pacemaker insertion. In Fig. 4, the top tracing showed his sinus bradycardia with premature atrial beats in 1969; a permanent pacemaker was installed at that time. By 1974, strips B and C indicated atrial fibrillation with a moderately fast heart rate. Carotid sinus massage, carried out beginning on the right side of strip B and continued into strip C, resulted in slowing of the ventricular response thereby allowing the demand pacemaker to initiate electrical impulses producing ventricular capture (strip C). Seventeen months later, the patient's pacemaker failed; strip $\mathrm{D}$ also obtained during carotid massage shows lack of ventricular capture by electrical stimulation (arrows) with atrial fibrillation which has remained stable for 18 months.

Case 9, age 80, showed sinus bradycardia with premature atrial beats in panel A of Fig. 5. A permanent demand pacemaker was inserted because of symptoms of SSS. Subsequently, a normally functioning pacemaker was temporarily inhibited by chest wall stimulation (Barold et al., 1970) thereby revealing underlying SSS (strips B and C). Five months later (strip D) the patient presented with atrial fibrillation and rapid ventricular response which suppressed the demand pacemaker. Though pacemaker failure occurred 6 months later, reinsertion was not recommended since the patient remained in stable atrial fibrillation without symptoms, which continues to be the case.

\section{(3) SSS WITH PERMANENT PACEMAKERS DEVELOPING ATRIAL FIBRILLATION AND INADEQUATE HEART RATE}

In only 1 of the 11 patients with SSS who developed stable atrial fibrillation was the ventricular response inadequate. This was an 83-year-old woman (Case 8 , Table 1) who presented at our institution in 1972 with atrial fibrillation and a very slow heart rate of

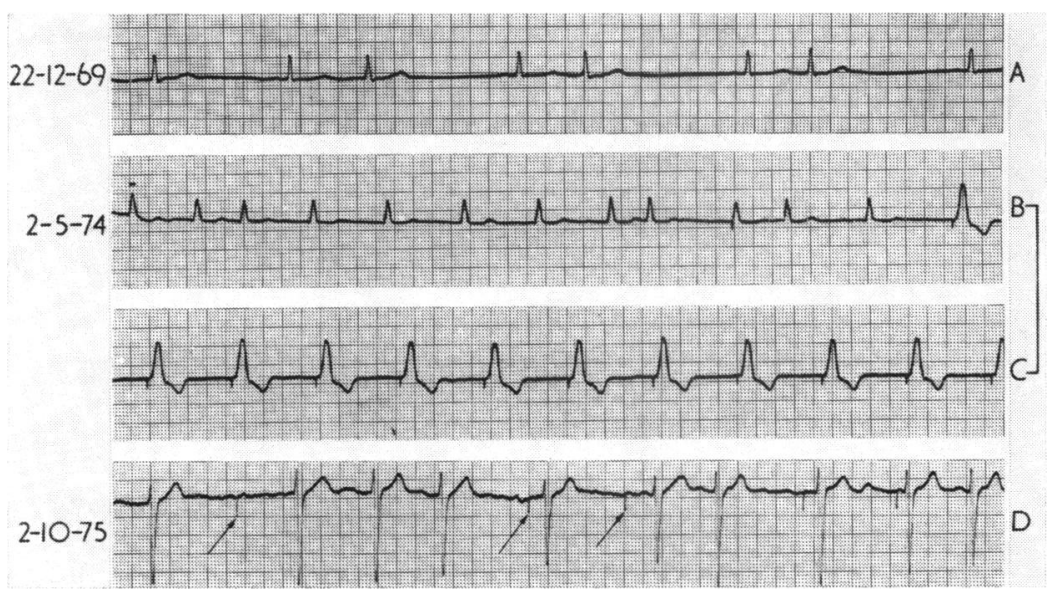

Fig. 4 Serial tracings in Case 4 with sick sinus syndrome who developed stable atrial fibrillation. Strip $A$ shows sinus bradycardia with frequent atrial beats. Strips $B$ and $C$ are continuous. Stable atrial fibrillation with normal demand pacemaker sensing is documented in strip $B$. In strip $C$ normal pacing function is unmasked by carotid sinus massage. In strip $D$ atrial fibrillation continues with a moderately slow ventricular response resulting from digitalis therapy and carotid massage; the pacemaker spikes (arrows) fail to capture the ventricle. 


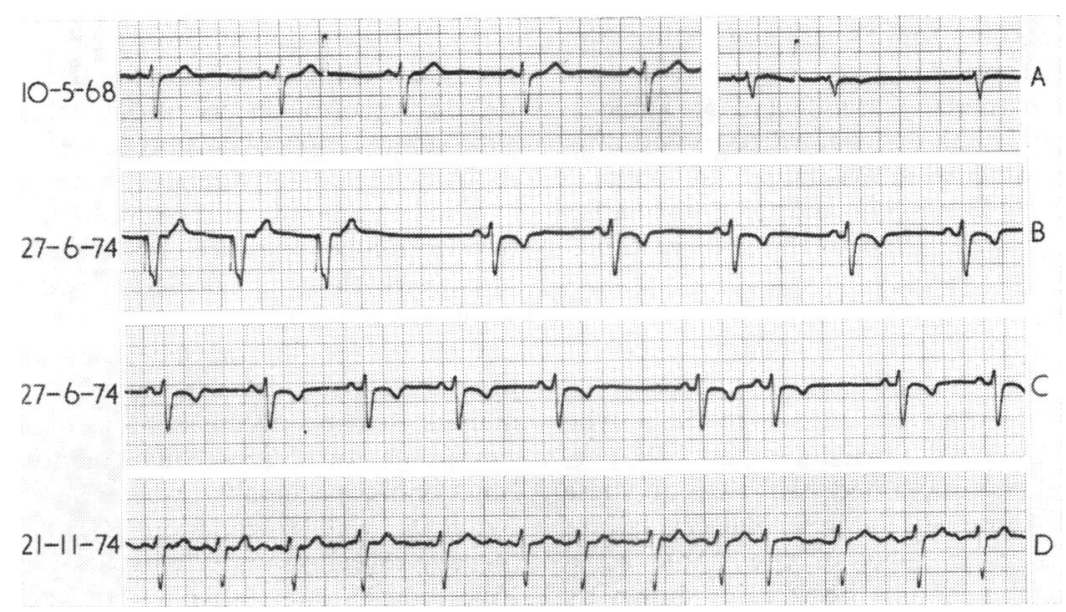

Fig. 5 Serial rhythm strips in Case 9 with SSS who developed stable atrial fibrillation. SSS was observed on the top panel $(A)$; subsequently a demand ventricular pacemaker was implanted. In the second panel $(B)$ ventricular pacing was temporarily inhibited after the first three beats by the method of chest wall stimulation which resulted in the emergence of SSS (strips $B$ and $C$ ). In the bottom panel $(D)$, a representative tracing showed stable atrial fibrillation with a moderately rapid ventricular response.

20 to $40 \mathrm{bpm}$ (Fig. 6). Because of repeated dizzy spells a permanent pacemaker was inserted. She had an 8-year history of lethargy and syncope. Previous electrocardiograms obtained from another hospital showed the onset of sinus bradycardia in 1963 and long periods of sinus arrest in 1968 (Fig. 6).

\section{Discussion}

The present study suggests that the development of stable atrial fibrillation in patients with the sick sinus syndrome (SSS) provides a spontaneous mechanism for the natural cure of the slow heart rate which occurs in this condition. For atrial

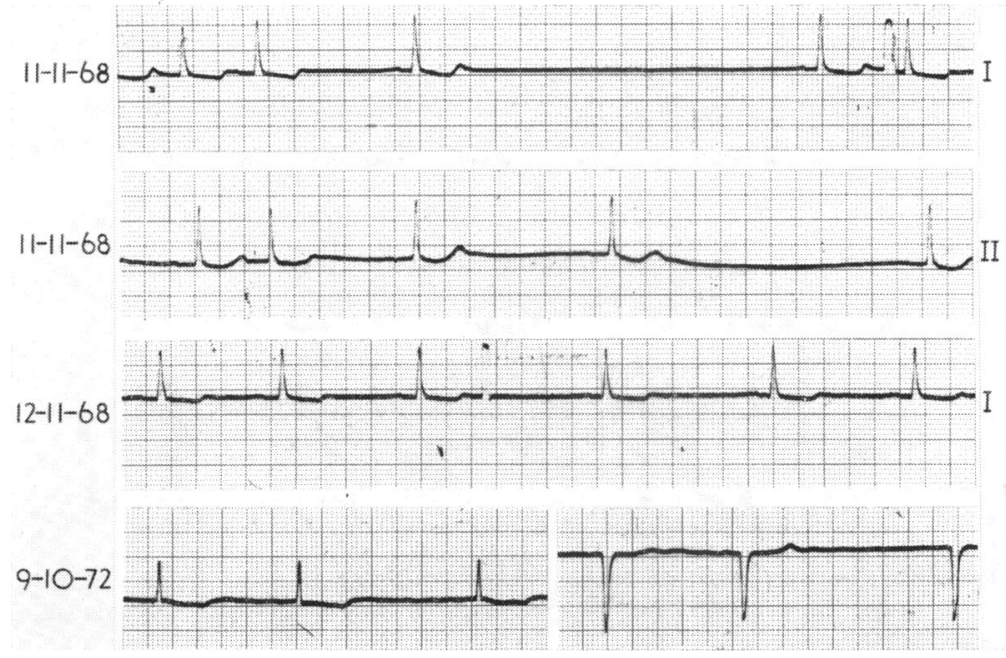

Fig. 6 Serial rhythm strips in Case 8 with sick sinus syndrome who developed chronic atrial fibrillation. The top three panels indicated periods of sinus arrest and premature atrial beats during which the patient experienced dizziness. The bottom panel shows stable atrial fibrillation but with a very slow ventricular rate of 20 to 40 bpm; her symptoms continued and a permanent demand ventricular pacemaker was inserted. 
fibrillation to afford a beneficial effect, this arrhythmia must be stable with an adequate ventricular response and should not compromise cardiac function or give rise to systemic embolisation. It is important to emphasise that there is an unsubstantiated clinical impression that atrial fibrillation occurring in SSS usually is associated with a very slow heart rate and so that this would be a continuing requirement for a permanent pacemaker. In this regard, our investigation provides evidence to the contrary since 10 of the 11 patients herein who developed stable atrial fibrillation manifested a perfectly adequate ventricular response (Table 1). Only one of our patients required a pacemaker for maintenance of a sufficient heart rate, whereas the remaining 10 continue to date to have clinically adequate ventricular responses. Indeed, 5 SSS patients with stable atrial fibrillation, 3 who had never undergone pacemaker insertion, and 2 with pacemaker failure, now have normal heart rates and remain entirely free of their symptoms which occurred regularly before the onset of atrial fibrillation.

The possibility that atrial fibrillation may appear in patients with sick sinus syndrome has been noted in reviews of this condition (Ferrer, 1973; Krishnaswami and Geraci, 1975). However, the occurrence of this arrhythmia was regarded only as an incidental observation; no special significance was attached to the development of atrial fibrillation in SSS, and information concerning the harbingers and clinical course of this rhythm alteration is lacking.

Our documentation of the development of stable atrial fibrillation in a substantial proportion of patients with SSS, which renders unnecessary the implantation of permanent pacemakers in nearly all of the patients in whom this occurs, raises certain important considerations: (1) elucidation of the factors predisposing to the development of atrial fibrillation in these patients; (2) clarification of the factors responsible for the persistence of atrial fibrillation; (3) identification of SSS patients likely to develop stable atrial fibrillation; (4) prediction of the onset of the arrhythmia in SSS; (5) delineation of the clinical course of atrial fibrillation seen in these patients; (6) determination of the haemodynamic consequences of atrial fibrillation in SSS ; and (7) examination of whether systemic embolisation constitutes a hazard with atrial fibrillation in this condition.

To provide the necessary information concerning these considerations, both retrospective and prospective assessment of the features of these patients was carried out. From this analysis, it was possible to determine a group of factors (Table 2) which
Table 2 Potential factors responsible for onset and persistence of stable atrial fibrillation in SSS

(1) Age of patient

(2) Type of associated heart disease

(3) Associated non-cardiac disease

(4) Severity of sinus bradycardia

*(5) Duration of symptomatic SSS

(6) Role of permanent pacemaker

*(7) Atrial enlargement

*(8) Intra-atrial conduction abnormalities

*(9) Previous atrial ectopy

*Denotes factors predisposing to stable atrial fibrillation in SSS.

appeared to be closely related to both the induction and persistence of atrial fibrillation in patients with the sick sinus syndrome. Of the potential factors examined, the duration of SSS symptomatology, the presence of intra-atrial conduction disorders, and previous atrial ectopy were each found to predispose to the subsequent development of chronic atrial fibrillation in SSS. Though the overall prevalence of radiographic or echographic atrial enlargement was infrequent in our SSS patients, 2 of the 4 patients with the finding developed stable atrial fibrillation. Features found to be unrelated to the occurrence of persistent atrial fibrillation in our population included patient age; associated heart disease such as coronary, hypertensive, or rheumatic cardiac involvement; and non-cardiac disorders such as chronic pulmonary disease and hyperthyroidism. In addition, neither the severity of sinus bradycardia nor the presence of a permanent pacemaker correlated with this condition.

In regard to the correlation of the duration of symptoms of SSS with the onset and perpetuation of atrial fibrillation, the patients developing stable atrial fibrillation had had symptomatic sinus bradycardia for 3 to 11 years (mean of 5.5 years) beforehand (Table 1). In contrast $(P<0.01)$, the SSS patients not developing atrial fibrillation had symptoms resulting from sinus bradycardia for only 0.8 to 3.5 years (mean of $2 \cdot 1$ years).

Intra-atrial conduction defects were present in 10 of the 11 patients developing atrial fibrillation (Table 1) compared $(P<0.01)$ with only 13 of the 45 SSS patients who remained in sinus rhythm. Disturbances of intra-atrial conduction were diagnosed by the presence of notched widened $(>2.5$ $\mathrm{mm}$ ) $\mathbf{P}$ complexes on limb leads I and/or II in the absence of radiographic or echographic left atrial enlargement.

Ectopic premature atrial beats were observed electrocardiographically in all 11 SSS patients before their subsequent development of stable atrial fibrillation (Table 1). Conversely $(P<0.01)$ ectopic premature atrial activity was noted in only 
21 of the 45 patients with SSS without chronic atrial fibrillation. These data indicate that ecoptic premature atrial systoles are consistent precursors of chronic atrial fibrillation in patients with sick sinus syndrome. The precipitation of atrial fibrillation by premature atrial beats is exemplified by a 46-year-old man who had SSS and episodic atrial flutter-fibrillation (Fig. 7). Brief periods of atrial fibrillation were regularly initiated by premature atrial contractions. Though persistent atrial activity is known to be associated with sinus bradycardia (Goel and Han, 1970), the mechanism of these atrial extrasystoles in SSS is largely speculative. However, their pathogenesis is consonant with the concept of atrial disease in patients with sick sinus syndrome (Ferrer, 1968). In agreement with this is the necropsy finding in SSS of diffuse fibrotic areas in the atria (Rubenstein et al., 1972) which may help to provoke ectopic atrial activity. In addition, this panatrial fibrosis may be responsible for the maintenance of atrial fibrillation. Furthermore, it is rational to assume that the tendency to develop atrial fibrillation increases with the gradual progression of atrial scarring in SSS. These observations are consistent with our findings in the present study that patients with long-standing symptomatic sinus bradycardia are particularly prone to the development of stable atrial fibrillation.

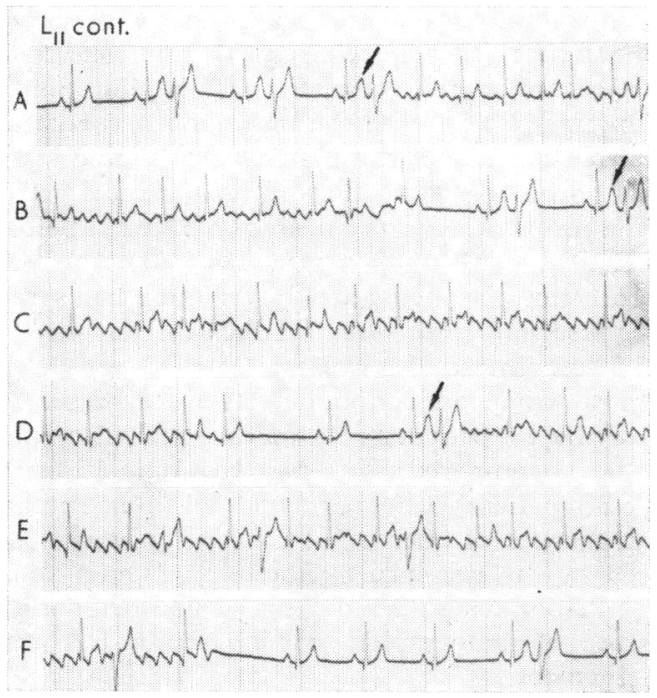

Fig. 7 Continuous recordings of limb lead II in patient having the sick sinus syndrome with paroxysmal atrial fibrillation. It is noteworthy that all episodes of atrial fibrillation were precipitated by premature atrial beats as seen in strips $A, B$, and $D$ (arrows).
The role of sinoatrial node re-entry in the genesis and maintenance of experimental supraventricular tachycardia has been established (Massumi et al., 1973). In view of the finding of delayed sinus node conduction time in SSS (Strauss et al., 1973), the possibility that a sinoatrial re-entry mechanism plays a part in the genesis of premature ectopic extrasystoles and atrial fibrillation in patients with the sick sinus syndrome requires consideration.

From the data presented here, patients with symptomatic sinus bradycardia for longer than 5 years appear to be likely to develop stable atrial fibrillation. In addition, SSS patients displaying premature atrial beats are especially prone to the onset of atrial fibrillation. The presence of both factors, long-standing SSS and premature atrial contractions, probably enhances the prediction of impending chronic atrial fibrillation. The clinical extension of the ability to anticipate generally the onset of stable atrial fibrillation in patients with SSS would be to withhold the insertion of permanent atrial pacemakers; demand ventricular pacemakers should be used instead. Since the development of atrial fibrillation in these patients would render inoperative both the sensing and pacing mechanism of atrial pacemakers, demand ventricular pacemakers would then be required for those with a slow ventricular response.

It is appropriate to emphasise that atrial fibrillation in SSS is often episodic. In this regard atrial fibrillation induced by atrial stimulation in an attempt to establish permanent atrial fibrillation in 3 SSS patients in sinus rhythm was unsuccessful because of reversion to slow sinus rate within 6 hours in each of these individuals. Therefore, atrial fibrillation should be considered stable only after serial 24-hour ambulatory electrocardiographic monitoring has established the persistence of this rhythm for at least 6 months. One of our 45 SSS patients without stable atrial fibrillation who had paroxysmal atrial fibrillation remained in atrial fibrillation for 3 months and then reverted spontaneously to a very slow sinus rate with associated syncope. Similarly, another SSS patient, a 57-yearold man, presented with syncopal episodes. His electrocardiogram showed alternating periods of atrial fibrillation and sinus bradycardia. Spontaneous termination of atrial fibrillation was occasionally associated with prolonged sinus arrest and cardiac asystole (Fig. 8).

While systemic embolisation is a potential complication of atrial fibrillation, none of our patients with SSS and chronic atrial fibrillation has had such an episode. Furthermore, in one large reported series of SSS patients, 4 of 50 patients who had systemic emboli were in sinus rhythm (Rubenstein 


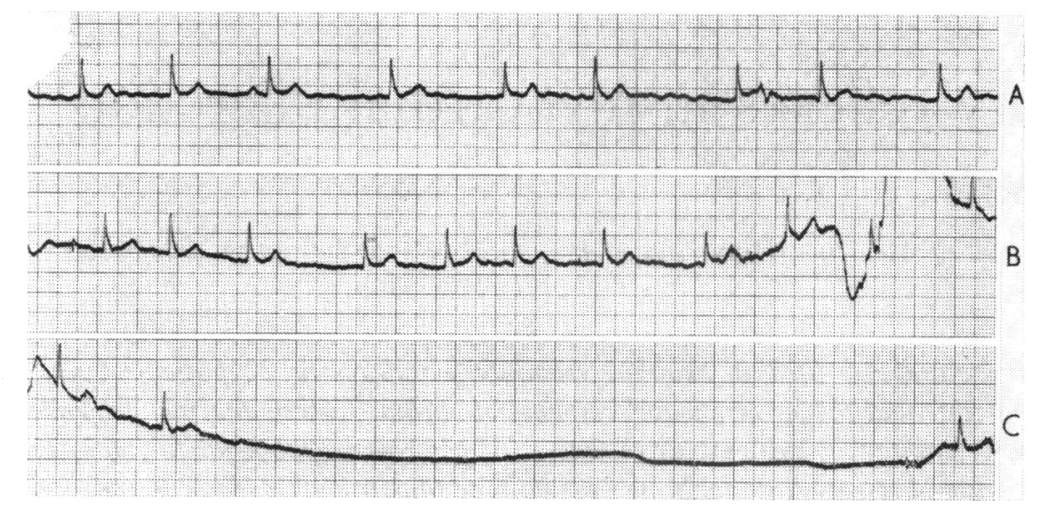

Fig. 8 Continuous lead II rhythm strips in a patient with SSS and episodic atrial fibrillation. Spontaneous termination of atrial fibrillation in strip $C$ was followed by a long pause of cardiac asystole lasting 8 seconds. Remarkably, no escape focus emerged during this prolonged period of sinus arrest.

et al., 1972). To date, systemic embolisation has not been encountered in any patient with SSS and stable atrial fibrillation. Therefore, it does not appear that SSS patients with atrial fibrillation have a special tendency for developing arterial emboli, and thus we do not recommend prophylactic anticoagulation in these patients.

It is concluded from the present investigation of a large group of SSS patients that: (1) development of stable atrial fibrillation in patients with SSS is not uncommon; (2) frequent periods of 24-hour ambulatory electrocardiographic monitoring should be undertaken to assess stability of atrial fibrillation; (3) permanent pacemaker therapy should not be discontinued unless persistent atrial fibrillation is present for more than 6 months; (4) in the absence of concomitant AV conduction disturbances, documentation of stable atrial fibrillation in SSS precludes the necessity of continued pacemaker therapy; and (5) the possibility of SSS should be considered in the elective cardioversion of atrial fibrillation so that one is prepared to treat any unmasked serious sinus bradyarrhythmia.

\section{References}

Barold, S. S., Pupillo, G., Gaidula, J. J., and Linhart, J. W. (1970). Chest wall stimulation in evaluation of patients with implanted ventricular inhibited demand pacemakers. British Heart fournal, 32, 783.

Ferrer, M. I. (1968). The sick sinus syndrome in atrial disease. Fournal of the American Medical Association, 206, 645.

Ferrer, M. I. (1973). The sick sinus syndrome. Circulation, 47, 635.

Goel, B. J., and Han, J. (1970). Atrial ectopic activity associated with sinus bradycardia. Circulation, 42, 853.

Krishnaswami, V., and Geraci, A. R. (1975). Permanent pacing in disorders of sinus node function. American Heart fournal, 89, 579.

Mandel, W., Hayakawa, H., Danzig, R., and Marcus, H. S. (1971). Evaluation of sinoatrial node function in man by overdrive suppression. Circulation, 44, 59.

Massumi, R. A., Fabregas, R., Tabaie, H., Zelis, R., Jewitt, M., Gray, D., and Mason, D. T. (1973). Reciprocation through the sino-atrial node. Clinical Research, 21, 238.

Rubenstein, J. J., Schulman, C. L., Yurchak, P. M., and DeSanctis, R. W. (1972). Clinical spectrum of the sick sinus syndrome. Circulation, 46, 5.

Short, D. S. (1954). The syndrome of alternating bradycardia and tachycardia. British Heart fournal, 16, 208.

Strauss, H. C., Saroff, A. L., Bigger, J. T., Jr., and Giardina, E. G. V. (1973). Premature atrial stimulation as a key to the understanding of sino-atrial conduction in man. Circulation, 47, 86.

Requests for reprints to Dr. Zakauddin Vera, Section of Cardiovascular Medicine, University of California at Davis, School of Medicine, Davis, California 95616, U.S.A. 